Communications in Physics, Vol.20, No. 4 (2010), pp. 349-358

\title{
SEARCH FOR HIGH ENERGY SKIMMING NEUTRINOS AT A SURFACE DETECTOR ARRAY
}

\author{
VO VAN THUAN, HOANG VAN KHANH, AND PHAM NGOC DIEP \\ Vietnam-Auger Cosmic Ray Laboratory
}

\begin{abstract}
In the present study we propose a new method for detection of high energy cosmological muon neutrinos by transition radiations at a medium interface. The emerging electro-magnetic radiations induced by earth-skimming heavy charged leptons are able to trigger a few of aligned neighboring local water Cherenkov stations at a surface detector array similar to the Pierre Auger Observatory. The estimation applied to the model of Gamma Ray Burst induced neutrino fluxes and the spherical earth surface shows a competitive rate of muon neutrino events in the energy range below the GZK cut-off.
\end{abstract}

\section{INTRODUCTION}

Along with the charged extremely high energy cosmic rays (EHECRs), the Pierre Auger Observatory (PAO) is able to detect very high energy neutrinos and the most perspective channel is searching by the surface Cherenkov detector array (SD) for earthskimming tauon neutrinos at energies from 0.2 to $20 \mathrm{EeV}\left(1 \mathrm{EeV}=10^{18} \mathrm{eV}\right)$ just below the region of Greisen-Zatsepin-Kuzmin (GZK) cut-off [1]. This detection window is available due to decays in flight of tau-leptons which produce very inclined air showers at low altitudes above the SD array. The horizontal events have been identified by a footprint geometry and by a timing criterion showing the signal transferring with the speed of light. The flash analogue-to-digital converter (FADC) signals of a SD stations caused by electromagnetic air showers can prove the origin of an event as coming from a cosmological neutrino.

Another complementary technique at PAO, the fluorescent detector telescopes (FD), can look for going-up or going-down air showers induced by both electron and tauon neutrinos. However, the FD window is for an order less effective [2] than the SD method. It was considered that muon neutrino was the worst flavor to be measured at PAO, either by SD array or FD telescopes. After more than one effective year of full operation, there was no tauon neutrino signal registered and an upper limit of tauon neutrino flux has been established [1].

In $[1,2]$ all three flavors of cosmological neutrinos are assumed to be in an equal ratio $1: 1: 1$ due to neutrino oscillation. Therefore, from the absence of tauon neutrinos one would extrapolate to the lack of muon and electron neutrinos. However, there are other models which consider the different proportions of neutrino flavors [3] which would introduce new physics. In this situation, in addition to tauon channel, a new detection window including muon neutrinos would be important for PAO. 
In the present work, instead of the decay-in-flight method [1], we look for the transition effect of electro-magnetic radiation induced at a medium interface by earth-skimming charged leptons and able to be detected at the PAO Cherenkov SD array. In the proposed method of detection we show that the emitted radiation from the earth surface can produce almost horizontal electromagnetic air showers of relative low energy, however, being just enough to trigger some few closest SD tanks. The plan of this article is the following: the flux of emerging heavy leptons is introduced in Sec. II. The detection probability of small inclined electromagnetic air showers at the Pierre Auger SD array and the rate of detectable events are estimated in Sec. III. The accidental coincidences and backgrounds are discussed in Sec. IV and the identification criteria are proposed in Sec. V.

\section{EARTH-SKIMMING NEUTRINOS AND EMERGING LEPTONS}

Cosmogenic neutrinos are believed to come from GZK effect of EHECRs just below the GZK-cutoff region [4] while the top-down models predict higher energy neutrinos [5]. The neutrino fluxes are being measured by AMANDA up to $0.0001 \mathrm{EeV}$ [6]. At very high energy above $0.001 \mathrm{EeV}$ neutrino fluxes are uncertain [7] and expected to decrease very fast by an exponential power law as $\Phi_{\nu}=\Phi_{0} E^{-\alpha}$, where $\alpha=2-3$. For further discussion the WB model in the frame of gamma ray burst mechanism (GRB) in [8] with $\Phi_{0}=4 \times 10^{-17} \mathrm{EeV}^{-1} \mathrm{~cm}^{-2} \mathrm{~s}^{-1}$ ster $^{-1}$ and with $\alpha=2$ is applied as a most available at neutrino energies between $0.001 \mathrm{EeV}$ and $10 \mathrm{EeV}$.

At energies higher $0.001 \mathrm{EeV}$, neutrino deep inelastic cross sections $\sigma_{\nu}$ increase with energy, due to which the Earth is getting more and more opaque for earth-skimming neutrinos. By the charge current interation (cc) we get electron and heavy leptons, muon or tauon. Electrons are easily to stop by bremsstrahlung in rock. Muons and tauons also loss their energy by radiative processes, however, they have a probability to survive and to emerge from the rock due to their relative longer radiative lengths. Based on the procedure described in [9] and [10] we calculated the fluxes of survived leptons. The rate of emerging leptons at a zenith angle and at a ceirtain energy [10] is as follows:

$$
\Phi_{L}\left(E_{L}, \theta\right)=\zeta \frac{N_{A}}{E_{L}+E_{C}} \int d E_{\nu} \Phi_{\nu} e^{-g} \int d y \frac{d \sigma^{c c}}{d y}\left[\frac{E_{L}}{E_{L}^{\prime}} \frac{E^{\prime}{ }_{L}+E_{C}}{E_{L}+E_{C}}\right]^{\eta},
$$

where $\zeta$ is a characteristic grammage of the radiative length of a skimming lepton. $E_{\nu}$ is neutrino energy. $E^{\prime}{ }_{L}$ and $E_{L}$ are initial and final lepton energies. $E_{C}$ is a critical energy above which radiative processes dominate the ionization. $N_{A}$ is Avogadro number. $\Phi_{\nu}$ is the cosmological neutrino flux. $g=g\left(x_{T}-x, E_{\nu}\right)$ is the exponential law of neutrino attennuation by deep inelastic interactions, where $x_{T}$ is the chord along which neutrino penetrates in the depth $x$. The chord can be expressed through the zenith angle, while the depth can be expressed through neutrino energy as:

$$
x=\zeta \times \ln \frac{E^{\prime}{ }_{L}+E_{C}}{E_{L}+E_{C}} .
$$

As $y$ is the inelasticity, i.e. the portion of netrino energy transferred to hadron component in a deep inelastic reaction, the lepton can get from neutrino another portion 
of energy as $E_{L}^{\prime}=E_{\nu} \times(1-y)$. The variable $y$ limits in:

$$
\begin{gathered}
y_{\min }=1 / E_{\nu} \max \left[E_{\nu}+E_{C}-\left(E_{L}+E_{C}\right) \times e^{-x_{T} / \zeta}, 0\right] \\
y_{\max }=1-E_{L} / E_{\nu} .
\end{gathered}
$$

The differential cross section depends on energy approximately as $d \sigma^{c c} / d y=\sigma_{0} E_{\nu}{ }^{0.363}$ as mentioned, where $\sigma_{0}=10^{-32} \mathrm{~cm}^{2}$ and $E_{\nu}=[E e V]$. The last term in (1) expresses the survival probability of lepton for emerging from the rock to the atmosphere, where: $\eta=m_{L} c^{2} \zeta /\left(E_{C} \rho \cdot c \tau_{L}\right)$, which contains lepton mass $m_{L}$, its lifetime $\tau_{L}$ and the density of standard rock $\rho=2.65 \mathrm{gr} \cdot \mathrm{cm}^{-3}$.

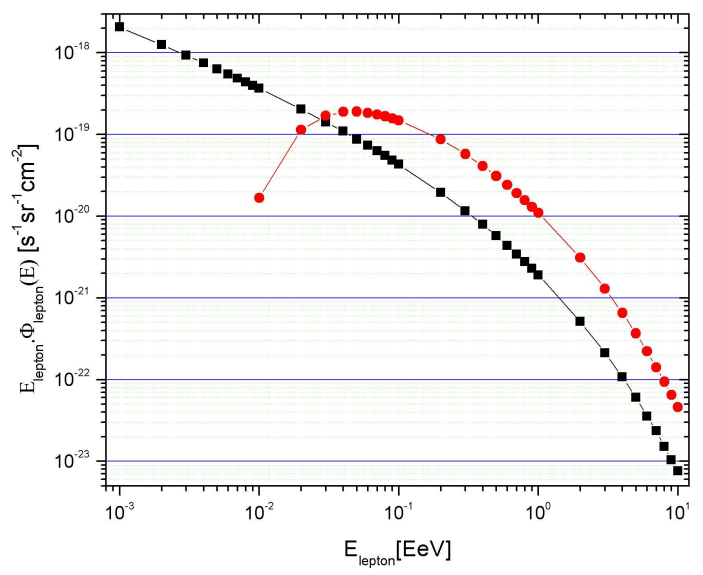

Fig. 1. Fluxes of emerging leptons

The physiscal process is quite similar for both muon and tauon, except the decay probability being more sensitive for tauon survival. In particular, at energies lower $0.01 \mathrm{EeV}$ tauons decay on a distance less than the radiative length and almost can not emerge from the rock, while it is not a problem for high energy muons to pass freely few radiative lengths without decay. The integration (1) gives us a flux of emerging leptons at a given energy range and under a given solid angle. In Fig. 1 there are shown graphs of estimated fluxes of muon and tauon in according to (1).

\section{THE DETECTION PROBABILITY AND THE RATE OF DETECTABLE EVENTS}

Skimming leptons interact with medium by bremsstrahlung (BS), direct pair production (DPP) and photonuclear reaction (PNR) [11]. At energies above $0.001 \mathrm{EeV}$, in case of muon the DPP dominates and consists of about 0.47 total contribution, while BS contribution is roughly 0.33 . PNR consists less 0.20 at lower $1 . E e V$ then getting more significant at energies higher 1 . EeV. At high energies above $0.001 \mathrm{EeV}$ PNR dominates for tauon which consists of 0.80 at 1 . EeV while DPP consists of 0.20 and BS is small. Photonuclear reaction cross section increases with energy and can not be useful for producing radiation at the medium interfasce. 
Most of radiative products are hardly to exit from the medium on several radiation lengths and their emerging radiations are usually ignored. Instead of this, in the present study we consider the residue at the low energy range of DPP and BS radiations which have a probability to exit from the earth surface in to the atmosphere. For both muon and tauon, for the first approximation we take in to account only contribution of DPP and BS, avoiding PNR, for which there is not a clear quantitative prediction at energies higher $0.001 \mathrm{EeV}[12]$.

For emerging from the surface, the relative intensity of electro-magnetic radiations (E-M) to be produced just in the thin layer of the radiative length of electron $L_{e}$ near to the surface is proportional to $L_{e} / L_{R}$, where $L_{R}$ is the radiative length of DPP+BS of muon or tauon. The cross sections of DPP as well as of the well-known BS are proportional to $1 / E_{R}$, where $E_{R}$ is the energy of radiation (electron-positron energy in case of DPP and $\mathrm{X}$-ray energy in case of $\mathrm{BS}$ ). In according to this law the radiation intensity is almost independent of energy. The emitted E-M radiation from the surface is proportional to the energy loss by skimming lepton in a thin layer of the earth surface of the order of a radiative length of electron as follows:

$$
E_{R}=\frac{1}{e} \times\left(L_{e} / L_{R}\right) E_{L}
$$

where $L_{e}=15 \mathrm{~cm}$ is radiative length of electron in rock; $L_{R}=9 \times 10^{4} \mathrm{~cm}$ and $5.6 \times 10^{5} \mathrm{~cm}$ are the radiative lengths by $\mathrm{DPP}+\mathrm{BS}$ of muon and tauon, respectively. For lepton energy between $0.001 \mathrm{EeV}$ and $10 . \mathrm{EeV}$, the intensity of E-M radiation is equivalent to an energy between roughly $5 \times 10^{10} \mathrm{eV}$ and $5 \times 10^{14} \mathrm{eV}$ at the medium surface.

In the atmosphere the emerging radiation with energies $10^{10} \mathrm{eV}-10^{15} \mathrm{eV}$ can produce moderate inclined E-M showers at a surface of the Cherenkov detector array of PAO.

For estimation of probability of those air showers to trigger some SD at the PAO array, we obtain the characteristics of the showers by the simulation based on the procedure similar to [13] and [14]. The simulations performed at five different energies give a linear dependence of the maximum depth $X_{\max }=[\mathrm{km}]$ on logarithm of shower energy $E_{A S}=$ $[e V]$ as shown in Fig. 2a.

The linear fit in Fig. 2 is approximate as:

$$
X_{\max }=2.10 \log \left(E_{A S}\right)-14.65 \text {. }
$$

As an example of simulation, the result of shower profile development at $2.8 \times 10^{12} \mathrm{eV}$ is described in Fig. 3. For defining the detection probability, the longitudinal size of a shower is roughly adopted as double of $X_{\max }$.

The laterial distribution and the transverse horizontal development of a shower is estimated by the mean square angular distribution of charged secondary particles [13] as: $<\theta>=0.85 \times E_{e}{ }^{-0.66}$, where the angle $\theta$ is given in radian; $E_{e}$ is energy of electron or positron, corresponding to the detection threshold of the PAO SD stations. Indeed, in [15] the SD array operating at a threshold of the FADC signal 2.4 VEM (Vertical Equvalent Muon) of the so-called ToT (Time-over-Threshold) which is a typical signal for electromagnetic showers. We adopt this value (equivalent to $640 \mathrm{MeV}$ ) as minimum energy of electron-positron to transfer to a SD station, of which the corresponding mean angle $\langle\theta\rangle=0.012$. Therefore, the horizontal transverse size $\langle r\rangle$ at maximum depth 


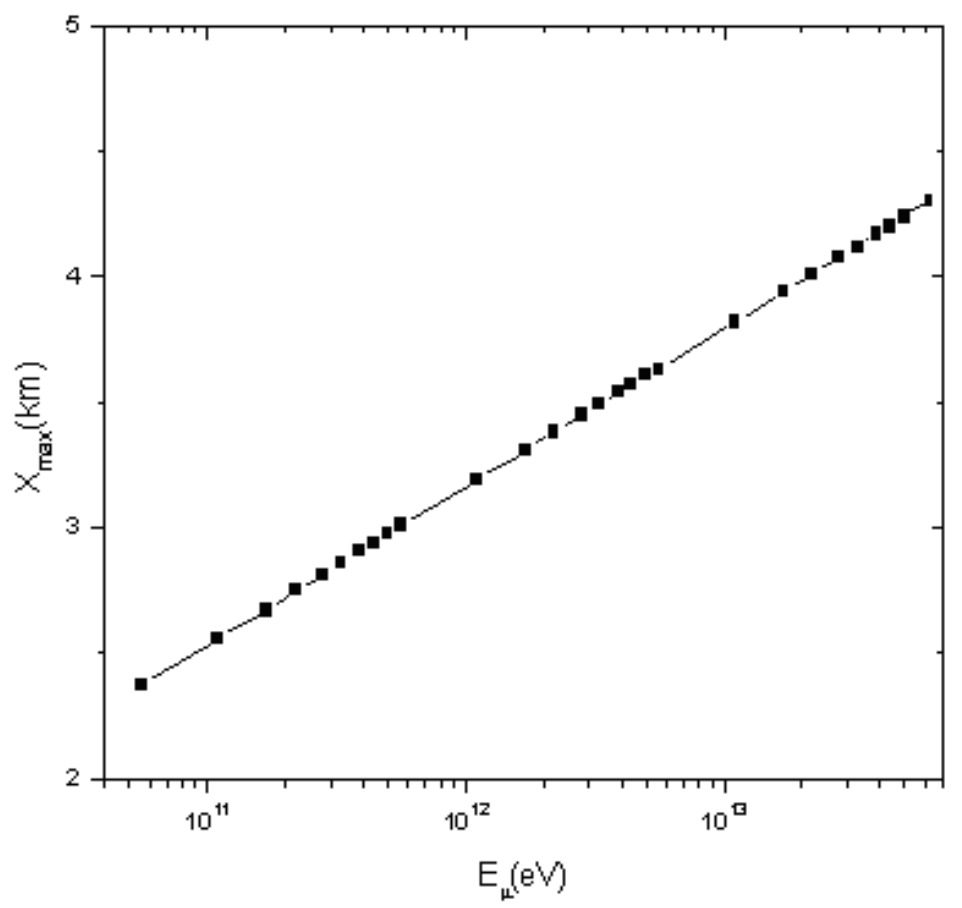

Fig. 2. Maximum depth vs. the shower energy.

$X_{\max }$ of a shower is estimated as:

$$
<r>=X_{\max } \tan \alpha_{\max }
$$

where $\alpha_{\max }=\sqrt{n_{\max }}\langle\theta\rangle$, while $n_{\max }$ is the number of radiative lengths until $X_{\max }$. This horizontal shower shadows the earth surface with an effective lozenge area as:

$$
S_{A S}=2 X_{\max }^{2} \tan \alpha_{e f f} .
$$

We expect that when the maximum depth $X_{\max }>2 \times L_{S D}=3.0 \mathrm{~km}$, i.e. twice larger than the effective distance $L_{S D}$ between two close SD tanks at PAO, the lozenge shadow covers at least a triplet of nearest SD stations and there is a probability to produce threefold ToT coincidences. In a simplified geometry, the probability of a shower covering and imprinting at the SD array producing three-fold coincidence is as:

$$
P_{A S}=2 \times \Delta \phi / 30^{0}=12 \times \alpha_{e f f} / \pi,
$$

where $\phi$ is azimuth.

The triggering probability of a tank depending on the energy density of secondary shower particles per $m^{2}$ is estimated as follows:

$$
P_{\text {Tank }}=2 N_{\text {Trigg }}{ }^{\text {max }} S_{\text {Tank }} /\left(\pi X_{\text {max }}^{2} \tan \alpha_{\text {eff }}^{2}\right),
$$

where the effective horizontal cross-section of a SD station $S_{\text {Tank }}=3.5 \mathrm{~m}^{2}$ and the intensity of secondary particles at $X_{\max }$ with an triggering energy $E_{\text {Trigg }}=2.4 \mathrm{VEM}$ is given 


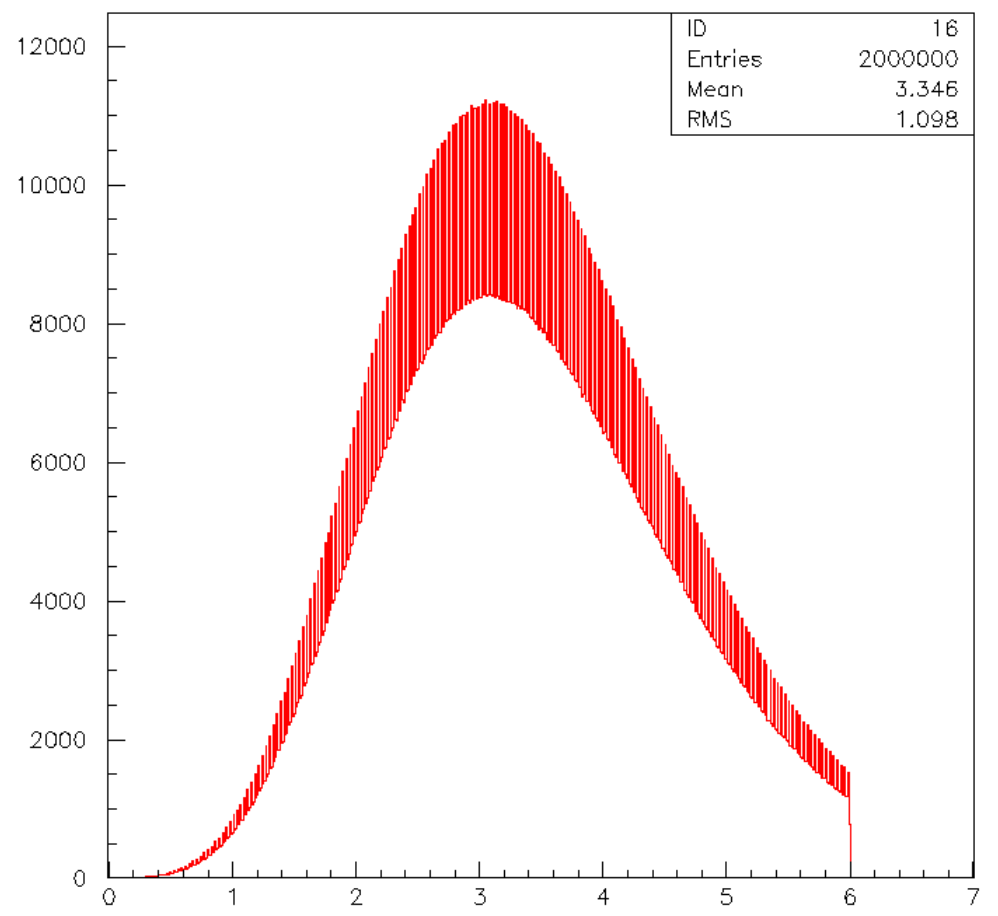

Fig. 3. Longitudinal development of E-M shower.

following [14]:

$$
N_{\text {Trigg }}{ }^{\text {max }}=0.31 .\left(E_{A S} / E_{\text {Trigg }}\right) / \sqrt{\ln \left(E_{A S} / E_{C}\right)}
$$

The effective angle $\alpha_{e f f}$ is calculated by the saturation condition when $P_{\text {Tank }}=1$. The effective aperture of the PAO site is obtained as:

$$
A_{\text {eff }}=P_{A S} P_{\text {Tank }}^{3} \times \pi \sin \alpha_{e f f^{2}} S_{P A O}=3.60 \times 10^{14} \alpha_{e f f}{ }^{3}\left[2-\left(\alpha_{e f f} / \alpha_{\max }\right)^{3}\right],
$$

where $S_{P A O}=3,000 \mathrm{~km}^{2}$ is PAO's square. The acceptance at a given shower energy is:

$$
A c c=A_{e f f} \times T .
$$

In Fig. 4 we show the energy dependence of acceptance where a saturation is happen at muon energy $E_{\text {muon }}>4 \mathrm{EeV}$, while for tauon the saturation has not been yet reached.

The acceptance being multiplified by the rate of emerging skimming leptons at a given energy gives the rate of detectable events per energy decade at PAO as follows [9]:

$$
N_{\text {Event }}=A c c\left[E_{L} \Phi_{L}\right] \ln 10
$$

In Fig. 5 we show the energy dependence of detectable rate of earth-skimming leptons, both muons and tauons. 


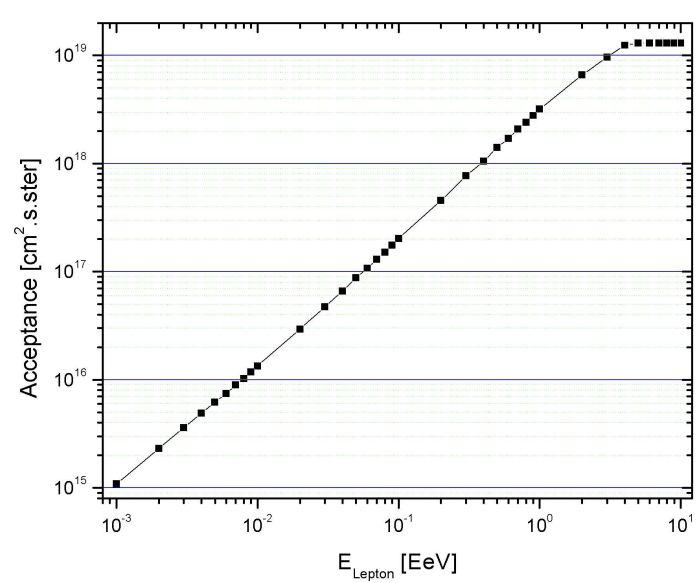

Fig. 4. The acceptance vs. muon energy

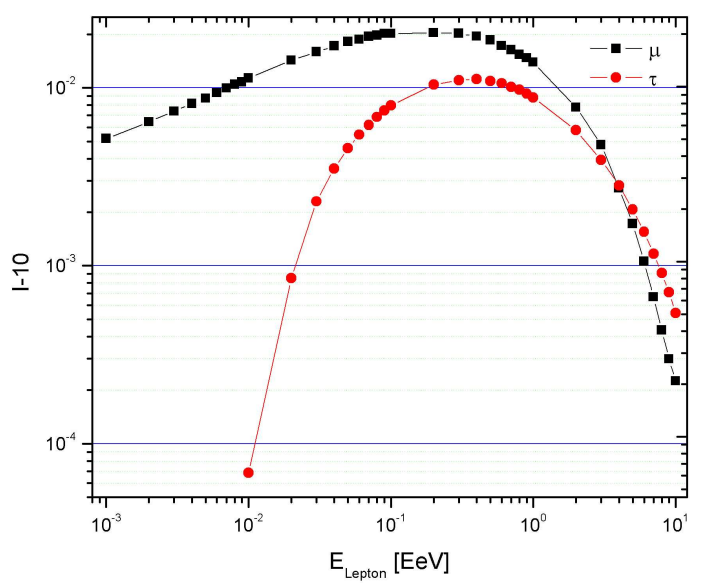

Fig. 5. Rates of detectable events

From this data we obtain the rate of detectable events for both muon and tauon neutrinos as shown in Table 1.

Table 1. Expected annual rate of detectable events at PAO (Southern site)

\begin{tabular}{cccccc}
\hline \hline Lepton energy decade (eV) & $(1-9)$. E15 & $(1-9)$. E16 & $(1-9)$. E17 & $(1-9)$. E18 & Integrated Rate \\
\hline Muon events & 0.0077 & 0.0150 & 0.0162 & 0.0034 & 0.042 \\
\hline Tauon events & $\sim 0.00$ & 0.0037 & 0.0091 & 0.0029 & 0.015 \\
\hline Muon+Tauon & 0.008 & 0.0187 & 0.0253 & 0.0063 & 0.057 \\
\hline \hline
\end{tabular}

\section{BACKGROUNDS}

The annual rate of three-fold ToT accidental coincidences of aligned tanks integrated over the PAO site is:

$$
N_{c}=(3 / 12)\left(2^{2} \Delta t^{2} N_{1} N_{2} N_{3}\right)\left(N_{S D} / N_{\text {Hexagon }}\right)(0.07)^{3} T,
$$

where the factor $3 / 12$ is the ratio of signals from aligned tanks to total number of three-fold signals in a hexagon. The rate of the second level (T2) trigger of a local station is $N_{i}=$ $20 \mathrm{~Hz}$ and the time window for two-fold coincidences is limited by $\Delta t=3 \mu \mathrm{sec}$ in according to the PAO technical instruction [15]. The third factor is the ratio of total number of tanks $N_{A S}=1600$ to the number of hexagons at the Southern site of $3,000 \mathrm{~km}^{2}$. Finally, the ratio between ToT electromagnetic signals and muon single-threshold signals is adopted equal 0.07 in according to the estimation in [16] and $T$ is the total year time in seconds. Therefore, we found the rate of the background of three-fold accidental coincidences per year is $N_{c}=2.3 \times 10^{-3}$, i.e. negligible.

Another source of background is the annual rate of three-fold ToT coincidences of aligned tanks induced by vertical air showers integrated over the PAO site which is estimated as:

$$
N_{c-A S}=(3 / 12)\left[(0.07)^{3}\left(\Delta t / \Delta t_{3}\right)^{3} N_{A S<3 E 17}\right]\left(S_{E-M} / S_{\mu}\right)^{2},
$$


where the second factor is a calibration of the effectiveness of Cherenkov tanks by counting the three-fold coincidences of air shower's ToT signals in the window $\Delta t$ instead of the large window $\Delta t_{3}=30 \mu \mathrm{sec}$, which have been given for the third level (T3) trigger, i.e. the central trigger of PAO and $N_{A S<3 E 17}=1.1 \times 10^{7}$ is the number of showers with energy less than the detection threshold $3 \times 10^{17} \mathrm{eV}$ which would be estimated by the data given in the PAO technical instruction [15]. The backgrounds from higher energy showers are always accompanied with strong air showers and easily to identify. The first factor and other values are the same as given in (12). The last factor is a geometrical ratio between the air shower's shadow of electromagnetic component and the shadow of muon component at the ground level as it is shown in Fig. 6.

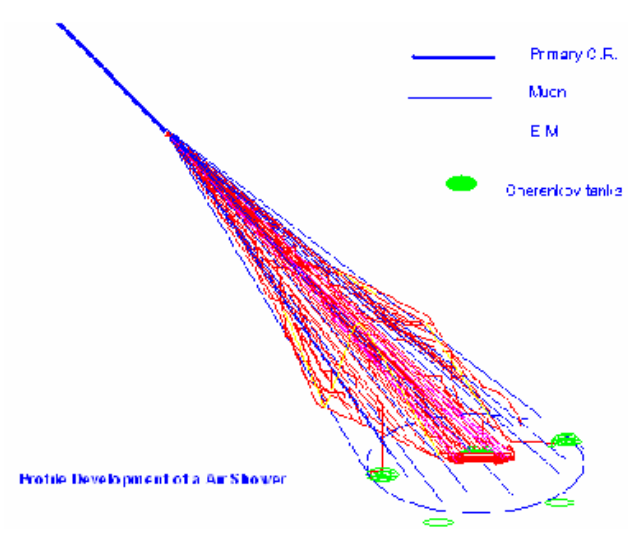

Fig. 6. The geometrical ratio between the shadows of electromagnetic component to muon one of a vertical air shower.

In Fig. 6 the radius of a shadow of E-M component at the ground is about 3 times narrower than the one of muon component. Therefore, two of three particles of a ToT three-fold coincidence should scatter at large angles to trigger the two neibouring tanks. The probability of those scattering effects is $P_{\text {scat }}=\left(S_{E-M} / S_{\mu}\right)^{2}=1 / 81$. Finally, the background of three-fold ToT coincidences from vertical air showers at the PAO detection threshold estimated by (13) is $N_{c-A S}=0.012$ event/year, which would be taken in to account of the total three-fold coincidence rate for identifying earth-skimming muon and tauon neutrinos in the energy range $0.003 \mathrm{EeV}-30 \mathrm{EeV}$.

Based on the event rate in Table 1 we found a ratio of signal-to-background $S / B<4.75$ which is available for identification.

\section{IDENTIFICATION CRITERIA}

The two- and three-fold coincidences induced by skimming muons or tauons should be around a hexagon, as the showers are low energy and not too extensive. The FADC traces should be of the time-over-threshold (ToT) type, i.e. electromagnetic shower signals as defined in [15]. They are expected moderate in intensity with the height of $2.4-$ 
6.0 VEM. A pair of FADC signals should follow a timing of transmission with a ground speed roughly equal to the speed of light, similar to the identification criterion adopted in [1]. Finally, if the sensitivity of SD stations allows to record signals below $0.2 \mathrm{VEM} /$ bin then we should look for possible electromagnetic residual appeared as under-threshold ToT signals at other SD tanks which are aligned on the same line of the three-fold triggered stations within a distance less than $10 \mathrm{~km}$. As more tanks give under-threshold ToT signals as stronger confirmation for a skimming neutrino induced event. The flavor ratio between muon and tauon events would be observed from the energy dependence of the event rate as shown in Fig. 5. However, due to a poor detectable rate in Table 1 it is hard to get a differential energy dependence. In the meantime, another independent detection method by decay-in-flight of tauons implemeted in [1] in combination with the currently proposed method, which is more effective for muon neutrino detection would allow to get the muon-tauon flavor ratio.

Almost all suggested criteria are able to be analized offline after triggering an electromagnetic (ToT) three-fold coincidence. The estimation given in Table 1 based on GRB model for the the Southern site of Pierre Auger project of the area $S_{P A O}=3,000 \mathrm{~km}^{2}$ shows a sensitivity of the new method as of roughly one event per life period (two decades) of the PAO. Another, Northern site of PAO is under construction in Colorado, USA which is expected at least twice larger and, therefore, would increase the sensitivity for detection rate of an event of earth-skimming muon neutrino per decade.

There are some sources of systematic uncertainties to study more as follows:

- This is to improve the estimation of propagation of neutrinos through rock medium which may shift the lepton energy spectra to lower direction.

- The uncertainty of geometry of air showers would contribute to systematical sources with a factor of $2-3$.

-The geographic topology of areas near to the PAO sites can contribute to horizontal zenith angle. For example, the Andes near to the Southern site can increase $\Delta \alpha_{\text {eff }}=0.01$.

\section{CONCLUSIONS}

We expect that the transition effects of DPP and BS radiations from a medium surface are induced by earth-skimming muons of high energies $0.001 \mathrm{EeV}-10 \mathrm{EeV}$ from cosmological neutrinos and then the moderate emitted radiations of roughly $10^{11}-10^{14} \mathrm{eV}$ can produce typical horizontal electromagnetic air showers to trigger few aligned neighboring SD tanks and can be detected by three-fold ToT coincidences. A similar but weeker detectable rate of tauon events is also expected.

\section{ACKNOWLEDGEMENT}

We thank Oscar Bigas Blanch (University of Paris 6, Jussieu) for introduction of their work on the earth-skimming tauon neutrino by decay-in-flight of tauons. Our present study is funded by the Ministry of Science and Technology (MOST) of Vietnam in the frame of the cooperative research project No.760/2008/HD-NDT for 2008-2009. 


\section{REFERENCES}

[1] Pierre Auger Collaboration,Phys. Rev. Let. 100 (2008) 211101.

[2] C. Aramo et al., Astropart. Phys. 23 (2005) 65.

[3] L. Anchordoqui et al., Astropart. Phys. 25(2006) 14.

[4] F. W. Stecker et al., Phys. Rev. Let. 66 (1991) 2697.

[5] Pierre Auger Collaboration, Astropart. Phys. 29 (2008) 243.

[6] AMANDA Collaboration, Astropart. Phys. 13 (2000) 1.

[7] T. K. Gaisser, F. Hazen, and T. Stanev, Phys.Rept. 258(1995)173.

[8] E. Waxman and J. N. Bahcall, Phys. Rev. D64(2001)023002.

[9] X. Bertou et al., Astropart. Phys. 17 (2002) 183.

[10] E. Zas, arXiv:, astro-ph/0504610v1(2005).

[11] S. I. Dutta et al., Phys. Rev. D63(2001)094020.

[12] R. P. Kokoulin and A. A. Petrukhin,Proc. of ICRC 6 (1971).

[13] J. W. Elbert et al.,Proc. of ICRC 27 (2001).

[14] A. M. Hillas, J. Phys. G: Nucl. Phys. 8 (1982) 1461.

[15] Pierre Auger Collaboration, Nucl. Inst. Meth. A523 (2004) 50.

[16] P. N. Dinh et al., Nucl. Phys. B627 (2002) 29.

Received 30 September 2009. 